\title{
Elitenstruktur und soziale Ungleichheit in Europa
}

Die Kluft zwischen Arm und Reich wird in Deutschland von Jahr zu Jahr größer. Die Diskussion über die Hintergründe dieser Entwicklung konzentriert sich bislang darauf, statistische Zahlen zu präsentieren bzw. zu interpretieren. Oder es geht um die Kritik an bestimmten Maßnahmen der Politik, vor allem in der Steuer- und der Sozialgesetzgebung. Außer Acht bleiben die Eliten als wesentliche Entscheidungsträger eines Landes. Ihre Rolle wird in diesem Beitrag näher beleuchtet. Dabei zeigt sich im Vergleich zwischen den einzelnen europäischen Ländern, dass es einen relativ direkten Zusammenhang zwischen der sozialen Exklusivität und Homogenität nationaler Eliten und dem Ausmaß der Einkommens- und Vermögensunterschiede gibt.

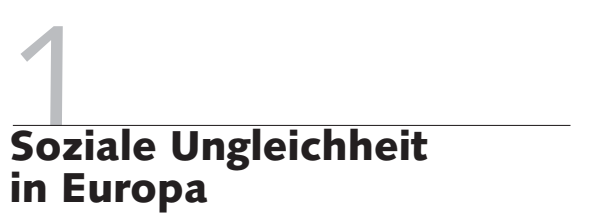

Wie krass die Schere zwischen Arm und Reich in Deutschland mittlerweile auseinander geht, zeigt ein plastisches Beispiel: Die 50 reichsten Deutschen haben ihr Vermögen im letzten Jahr um 50 Mrd. $€$ vermehren können (Mattheis 2007). Von dieser Summe müssten sich die knapp zwei Mio. Kinder, die von Hartz IV leben müssen, bei einem Verpflegungssatz von täglich $2,29 €$, über 30 Jahre lang ernähren. Statistische Zahlen belegen diese Entwicklung ebenfalls deutlich. Seit 1998 ist der Anteil der Armen an der bundesrepublikanischen Bevölkerung von gut 12 auf über $17 \%$ gestiegen. Gleichzeitig nahm das Nettovermögen der Bundesbürger allein zwischen 1998 und 2003 um $17 \%$ auf insgesamt 5 Billionen $€$ zu. Die Verteilung dieses Vermögens fällt allerdings höchst ungleich aus. Die vermögendsten $10 \%$ der Bevölkerung besitzen mittlerweile nicht nur mehr als die Hälfte dieses Vermögens, sie haben ihren Anteil seit 1998 auch noch einmal deutlich erhöhen können. Die ärmsten $10 \%$ haben ebenfalls eine Steigerung zu verzeichnen: Ihre Schulden haben sich allein bis 2003 verdreifacht; Nettovermögen besitzen sie keines. Die gesamte untere Hälfte der Bevölkerung verfügt noch nicht einmal über $4 \%$ des Nettovermögens der Bundesbürger. Bei den Einkommen lässt sich, wenn auch in weniger scharfer Form, derselbe Trend beobachten. Die oberen $10 \%$ haben ihren Anteil am Gesamteinkommen zwischen 1995 und 2003 um ein gutes Zehntel von 29,7 auf 33,3\% steigern können, das oberste Prozent sogar um mehr als ein Fünftel von 9 auf 10,9\% (Bundesministerium für Gesundheit und Soziale Siche-

\begin{tabular}{|c|c|c|c|c|}
\hline & $\begin{array}{l}\text { Einkommens- } \\
\text { relationen: } \\
\text { oberstes zum } \\
\text { untersten Fünftel }\end{array}$ & $\begin{array}{l}\text { Relationen der } \\
\text { Löhne: } \\
\text { oberstes zum } \\
\text { untersten Zehntel }\end{array}$ & $\begin{array}{c}\text { Gini-Koeffizient } \\
\text { - in \% - }\end{array}$ & $\begin{array}{l}\text { Armutsquote } \\
- \text { in } \%-\end{array}$ \\
\hline \multicolumn{5}{|l|}{ Skandinavien } \\
\hline Dänemark & 3,4 & 2,1 & 0,24 & 11 \\
\hline Finnland & 3,5 & 2,0 & 0,25 & 11 \\
\hline Norwegen & 3,6 & 2,0 & $0,26^{*}$ & 11 \\
\hline Schweden & 3,3 & 2,0 & 0,23 & 11 \\
\hline \multicolumn{5}{|l|}{ Osteuropa } \\
\hline Bulgarien & 4,0 & 4,4 & $0,26^{*}$ & 15 \\
\hline Polen & $5,0^{*}$ & 4,7 & $0,31 *$ & 18 \\
\hline Rumänien & $4,6^{*}$ & 5,1 & $0,31^{*}$ & 18 \\
\hline Tschechien & $3,4^{*}$ & 2,8 & $0,24^{*}$ & 9 \\
\hline Ungarn & $3,3^{*}$ & 3,4 & $0,23^{*}$ & 12 \\
\hline \multicolumn{5}{|l|}{ Mitteleuropa } \\
\hline Belgien & 4,0 & 2,6 & 0,26 & 15 \\
\hline Deutschland & 4,4 & 3,2 & 0,28 & 16 \\
\hline Niederlande & $4,0^{*}$ & 2,8 & $0,27^{*}$ & 12 \\
\hline Österreich & 3,8 & 3,0 & 0,26 & 13 \\
\hline \multicolumn{5}{|l|}{ Westeuropa } \\
\hline Frankreich & 4,2 & 3,4 & 0,28 & 13 \\
\hline Großbritannien & $5,3^{*}$ & 3,2 & $0,35^{*}$ & 19 \\
\hline Irland & 5,0 & 3,9 & 0,32 & 21 \\
\hline \multicolumn{5}{|l|}{ Südeuropa } \\
\hline Griechenland & 6,0 & 2,6 & 0,33 & 20 \\
\hline Italien & 5,6 & 2,5 & 0,33 & 19 \\
\hline Portugal & 7,2 & 3,3 & 0,38 & 21 \\
\hline Spanien & 5,1 & 3,2 & 0,31 & 20 \\
\hline
\end{tabular}

rung 2005, S. 18ff.; Grabka/Frick 2007, S. $667 \mathrm{ff}$; Groh-Samberg 2007, S. 177ff.; SPD-Bundestagfraktion 2007, S. 105).

In einer ganzen Reihe der europäischen Länder sieht die generelle Entwicklung ähnlich aus. Was die konkrete Verteilung von Armut und Reichtum angeht, gibt es allerdings von Land zu Land große Unterschiede (Tabelle 1). So liegt die Armutsquote in Großbritannien, Portugal oder Spanien bei ca. $20 \%$. Sie ist fast doppelt so hoch wie die der Tschechischen Republik und der skandinavischen Staaten. Bei alleinstehenden Personen mit versorgungsbedürftigen Kindern liegen die Werte für
Deutschland, Großbritannien und Irland mit 44 bis $54 \%$ sogar ungefähr dreimal so hoch wie in Dänemark, Finnland, Luxemburg, Schweden und Ungarn mit 15 bis 19 $\%$ (Liddle/Lerais 2006, S. 55). Beim Reichtum ist es genau umgekehrt. Das oberste Fünftel der Bevölkerung vereint in Portugal

\footnotetext{
Michael Hartmann, Prof. für Soziologie, TU Darmstadt. Arbeitsschwerpunkte: Eliten-, Management- und Bildungssoziologie im internationalen Vergleich. e-mail: hartmann@ifs.tu-darmstadt.de
} 
ein mehr als sieben Mal höheres Einkommen auf sich als das unterste Fünftel, in Großbritannien und Spanien ein mehr als fünf Mal höheres. In den skandinavischen Ländern und der Tschechischen Republik ist es dagegen „nur“ das 3,3 bis 3,6-Fache.

\section{Die Rolle der Eliten}

Angesichts der immer weiter zunehmenden Kluft zwischen Reich und Arm in Europa stellt sich die Frage, inwieweit die Eliten diese Entwicklung spürbar beeinflussen oder eventuell sogar steuern können. Die beträchtlichen Differenzen zwischen den einzelnen europäischen Staaten, soweit es die Verteilung der jeweiligen nationalen Einkommen und Vermögen angeht, zeigt eines deutlich: Auch wenn die Eliten all dieser Länder bestimmten Rahmenbedingungen unterliegen (wie beispielsweise der Finanzmarktsituation oder der Finanzpolitik), die sie durch ihre Entscheidungen nur teilweise bestimmen können, so bleiben ihnen doch ganz offensichtlich nicht unerhebliche Handlungsspielräume.

Typisch ist die aktuelle Diskussion über die Rolle von Private Equity und Hedgefonds oder die Steuerpolitik. Politiker wie Topmanager weisen immer wieder darauf hin, dass sie selbst Getriebene der Entwicklung seien. Der Druck der Finanzmärkte, so das stets wiederkehrende Argument, lasse ihnen einfach keine Wahl. Eine solche Argumentation ist nicht einfach von der Hand zu weisen. Spektakuläre Einflussnahmen von Hedgefonds, wie im Falle der Deutschen Börse, zeigen das ebenso wie die bei multinationalen Konzernen übliche Verschiebung von Gewinnen in steuergünstige Länder. Unter kapitalistischen Bedingungen werden von Menschen geschaffene Verhältnisse zu äußeren, scheinbar naturgesetzlichen Zwängen, denen sich auch Topmanager oder Spitzenpolitiker nicht einfach entziehen können.

Das ist aber nur die eine Seite der Medaille. Wer mit dem Druck der Finanzmärkte argumentiert, sollte nicht vergessen, dass politische Entscheidungen zur Deregulierung dieser Märkte geführt haben. Die Deregulierung war nicht einfach „naturgesetzlich“ vorgegeben. Das gilt vor allem hinsichtlich ihrer konkreten Form und ihres Ausmaßes, und zwar bis heute, wie die Debatte um die Kontrolle von
Hedgefonds unmissverständlich demonstriert. Zudem war und ist der schnelle und umfassende Erfolg dieser Fonds untrennbar verknüpft mit der stark steigenden Zahl an Multimillionären, die lukrative Anlagemöglichkeiten für ihr Geld suchen. Er steht also in direktem Zusammenhang mit der wachsenden Spaltung der Gesellschaft in Arm und Reich, und Letzteres wiederum mit politischen Entscheidungen, vor allem in der Steuerpolitik.

Gerade die deutschen Finanzminister sind in den letzten 15 Jahren nicht müde geworden, auf externe Zwänge hinzuweisen, die sie zu einer nachhaltigen Senkung der Spitzensteuersätze oder zum Verzicht auf eine Vermögenssteuer nötigten. Sie zelebrierten sich als Getriebene einer Entwicklung, waren in Wirklichkeit aber häufig eher die Treibenden. Aktuelle Vergleiche der effektiven Steuersätze, die vom Bundesfinanzministerium regelmäßig veröffentlicht werden, verdeutlichen das anschaulich. Die Steuerquote lag in Deutschland schon 1995 deutlich unterhalb des Niveaus der meisten Industrieländer. In Europa wiesen nur Spanien und die Schweiz niedrigere Quoten auf. Auch bei Berücksichtigung der Sozialabgaben ändern sich die Verhältnisse nicht wesentlich. Die Unterschiede werden allerdings geringer. Akuter, von außen erzwungener Handlungsbedarf war und ist dennoch nicht $\mathrm{zu}$ erkennen. Man hat die Steuersätze trotzdem deutlich gesenkt. Deutschland weist mittlerweile eine Steuerbelastung von nur noch $20,8 \%$ auf, ${ }^{1}$ während in den meisten anderen Ländern die Quote nicht nur gleich geblieben ist, sondern sich vielfach sogar noch erhöht hat. Bei der Gesamtbelastung inklusive der Sozialabgaben zeigt sich dasselbe Bild. Sie ist hierzulande von 37,2 auf nur noch $34,7 \%$ gesunken, während sie in den meisten anderen Ländern leicht (auf Werte zwischen knapp 40 und gut $50 \%$ ) gestiegen ist. Selbst Spanien hat mit 35,8 \% inzwischen eine höhere Abgabenquote als Deutschland zu verzeichnen (Bundesministerium der Finanzen 2005, S. 8f., 2007, S. 56). Nach einem Jahrzehnt Steuerreformen liegt Deutschland unter den Industriestaaten in puncto Steuerquote am unteren Ende der Skala und hinsichtlich der gesamten Abgabenlast im unteren Drittel.

Eine OECD-Studie macht auf einen weiteren entscheidenden Aspekt aufmerksam. Die Steuerreformen in Deutschland, so die Kernaussage, hätten eine drastische soziale Schieflage zur Folge. Entlastet worden seien in erster Linie die gut verdienenden Angestellten, Freiberufler und Unternehmer. ${ }^{2}$ Überhaupt nicht entlastet, sondern sogar stärker als zuvor belastet worden seien dagegen alleinerziehende Geringverdiener. Eine alleinerziehende Mutter mit zwei Kindern und rund zwei Dritteln des Durchschnittseinkommens müsse mit gut $21 \%$ heute um ein Fünftel höhere Abgaben entrichten als im Schnitt der OECD-Länder (vgl. Dunkel 2007). Die im europäischen Vergleich sehr hohe Armutsquote für diese Personengruppe resultiert zu einem beträchtlichen Teil aus der Steuerpolitik der letzten Bundesregierungen.

Von Sachzwängen kann also nicht die Rede sein. Die Eliten sind keine quasi neutralen Sachwalter externer Zwänge, sie verfolgen durchaus gezielt eigene Interessen.

\section{Eliten und gesellschaftli- che Kräfteverhältnisse}

In welchem Ausmaß ihnen die Durchsetzung ihrer Interessen gelingt, hängt vor allem von zwei Faktoren ab: der Homogenität der zentralen Eliten zum einen sowie den gegebenen gesamtgesellschaftlichen Kräfteverhältnissen zum anderen. Eliten zeichnen sich aus durch eine gehobene soziale Herkunft aus Bürger- oder Großbürgertum, den gemeinsamen Besuch exklusiver Bildungsinstitutionen sowie den regelmäßigen Wechsel zwischen Spitzenpositionen in den verschiedenen zentralen Sektoren; sie sind also in ihren Einstellungen und Interessen relativ homogen. Es dürfte auf der Hand liegen, dass sie damit auch bei der Interessendurchsetzung erfolgreicher sind als diejenigen, die aus anderen, heterogeneren Gesellschaftsschich-

\footnotetext{
Diese Zahlen beziehen sich auf das Jahr 2005.

2 Allen offiziellen Begründungen für die Senkung des Spitzensteuersatzes zum Trotz könnte die ganz überwiegende Mehrzahl dieser Personen die steuerlichen Möglichkeiten eines Wohnortwechsels ins Ausland im Übrigen gar nicht nutzen. Weder die höheren Beamten noch die niedergelassenen Ärzte, Apotheker und Anwälte oder die durchschnittlichen leitenden Angestellten und Mittelständler können Deutschland einfach verlassen. Sie sind beruflich zumeist auf die (steuerlich relevante) überwiegende Anwesenheit in Deutschland angewiesen
} 
ten und -klassen stammen, relativ weitverbreitete Bildungstitel aufweisen und ihre gesamte Karriere in nur einem Bereich absolvieren. Die Realisierung der Elitenziele hängt allerdings von den je nach Land und historischer Phase unterschiedlich starken Gegenkräften ab. Entscheidend sind in dieser Beziehung drei Punkte: erstens die in der breiten Bevölkerung verankerten Wertvorstellungen und die generell vorhandene Bereitschaft zum Widerstand; zweitens die dauerhafte Organisierung gegenläufiger Interessen vor allem in Form von Gewerkschaften; und drittens das Verhältnis der breiten Bevölkerung zu den parlamentarischen Möglichkeiten der Einflussnahme, d.h. zu Wahlen und Parteien.

Betrachtet man die Eliten in Europa, so lassen sich vier Grundtypen von Elitenbildung und Elitenhomogenität erkennen. Es gibt erstens Eliten mit einer einheitlichen Ausbildung in Elitebildungseinrichtungen, einer in allen Bereichen durchgängig hohen sozialen Rekrutierung aus Bürger- und Großbürgertum und zusätzlich auch noch einer hohen sektorübergreifenden Mobilität (Frankreich). Zweitens gibt es Eliten, die zwar eine ähnlich exklusive soziale Herkunft quer durch alle Bereiche aufweisen, jedoch entweder intersektoral nicht mobil sind (Großbritannien) oder aber keine Elitebildungsstätten besucht haben (Spanien, Portugal). Drittens gibt es Eliten, die weder mobil noch in Eliteinstitutionen ausgebildet sind, sozial aber, vor allem soweit es die Wirtschaft betrifft, ebenfalls vom Nachwuchs des Bürger- und Großbürgertums dominiert werden (Deutschland, Italien, Niederlande). Deutschland ähnelt aufgrund der bürgerlichen Prägung, die auch die Spitzen in Verwaltung und Justiz charakterisiert, sowie der in den letzten Jahren beobachtbaren Verbürgerlichung der politischen Elite dabei Frankreich, Großbritannien und Spanien am stärksten. Viertens schließlich gibt es Eliten, die nicht nur die normalen Bildungseinrichtungen absolviert und ihre Karrieren fast ausschließlich in einem Sektor gemacht haben, sondern auch sozial erheblich durchlässiger sind als die schon genannten Eliten (Skandinavien). Die übrigen Länder bilden entweder Mischformen aus den Typen drei und vier oder sie sind (wie die neuen EU-Mitglieder in Osteuropa) noch dabei, eine spezifische Form von Elitenbildung zu entwickeln (Hartmann 2007, S. 83ff.).

In Hinblick auf die Homogenität der Eliten bilden diese vier Typen zugleich eine

\begin{tabular}{|c|c|c|c|c|}
\hline & Großbürgertum & Bürgertum & Mittelschichten & Arbeiterklasse \\
\hline Frankreich & 52,5 & 27,5 & 15,0 & 5,0 \\
\hline Großbritannien & 45,5 & 27,2 & 18,2 & 9,1 \\
\hline Spanien & 40,0 & 20,0 & 40,0 & - \\
\hline Portugal & 22,2 & 66,7 & 11,1 & - \\
\hline Deutschland & - & 31,2 & 43,8 & 25,0 \\
\hline Italien & 8,7 & 39,1 & 30,4 & 21,7 \\
\hline Niederlande & 23,0 & 15,4 & 30,8 & 30,8 \\
\hline Belgien & 28,6 & 21,4 & 28,6 & 21,4 \\
\hline Österreich & - & 12,5 & 37,5 & 50,0 \\
\hline Schweiz & 28,5 & 14,3 & 42,9 & 14,3 \\
\hline Dänemark & - & 15,4 & 53,8 & 30,8 \\
\hline Finnland & 20,0 & - & - & 80,0 \\
\hline Norwegen & 15,4 & 15,4 & 46,1 & 23,1 \\
\hline Schweden & 22,2 & 11,1 & 33,3 & 33,3 \\
\hline Polen & - & 66,7 & - & 33,3 \\
\hline Ungarn & - & 40,0 & 20,0 & 40,0 \\
\hline
\end{tabular}

Hierarchie, an deren Spitze die französischen Eliten als die homogensten und zugleich am besten verankerten stehen, dicht gefolgt von den britischen, portugiesischen und spanischen. Dann folgen (mit mehr oder minder großem Abstand) die deutschen, italienischen und niederländischen Eliten. Und am Ende dieser Skala rangieren als die sozial zugänglichsten und heterogensten die Eliten aus Skandinavien und einigen Staaten in Osteuropa.

Die übrigen europäischen Länder unterscheidet von Frankreich, Großbritannien, Portugal und Spanien eine erheblich breitere soziale Rekrutierung der Eliten. Das gilt vor allem für die Politik. Während sich die Eliten in den erstgenannten vier Staaten ganz überwiegend aus dem Bürger- und Großbürgertum rekrutieren, besetzen in den übrigen Ländern traditionell Mittelschicht- und Arbeiterkinder die Mehrzahl der politischen Spitzenpositionen. Am stärksten sind Letztere in Skandinavien und Österreich vertreten (Tabelle 2).

In Deutschland und Italien, den beiden einzigen großen westeuropäischen Staaten mit einer traditionell relativ offenen politischen Elite, ist in den letzten Jahren allerdings ein neuer Trend zu erkennen. Die Verankerung der Parteien in der Bevölkerung nimmt drastisch ab, der Anteil der Bürger- und Großbürgerkinder in den Regierungen gleichzeitig erheblich zu. Am deutlichsten wird das beim Vergleich der großen Koalitionen unter Kiesinger und Merkel. Während das Bundeskabinett vor 40 Jahren noch zu fast zwei Dritteln von
Politikern gebildet wurde, die in den breiten Mittelschichten oder der Arbeiterklasse aufgewachsen waren, liegt ihr Anteil heute nur noch bei gut $30 \%$. Umgekehrt stellen Großbürgerkinder, die damals überhaupt nicht vertreten waren, heute jeden achten Minister und Bürgerkinder über die Hälfte statt einem guten Drittel der Kabinettsmitglieder. Die Bundesregierung hat sich in ihrer sozialen Zusammensetzung den Regierungen Frankreichs, Großbritanniens und Spaniens stark angenähert. In Italien ist die Entwicklung in den letzten 15 Jahren ähnlich verlaufen. Ein Verbürgerlichungsprozess der politischen Eliten bei gleichzeitig massivem Verlust an Parteimitgliedern ist auch in Belgien, den Niederlanden und selbst den skandinavischen Staaten zu beobachten. Von den Kabinettsmitgliedern der aktuellen großen Koalitionen in den Niederlanden und Österreich kommen im Unterschied zu Deutschland allerdings immer noch gut $60 \%$ aus der breiten Bevölkerung.

In der Wirtschaft sieht es generell etwas anders aus. Die Topmanager in Deutschland und Italien rekrutieren sich zu einem fast identischen Prozentsatz wie die in Frankreich, Großbritannien oder Spanien aus dem Großbürgertum. Berücksichtigt man noch den Nachwuchs des Bürgertums, existiert zwischen Deutschland und diesen drei Ländern gar kein Unterschied mehr. Die italienische Wirtschaftselite dagegen ist mit einem gut doppelt so hohen Prozentsatz an Managern aus den Reihen der Mittelschichten und der Arbeiterklasse sozial zumindest etwas zugänglicher. Deut- 


\begin{tabular}{lcccccc}
\hline \multicolumn{1}{l}{ Tabelle 3: Die soziale Rekrutierung der Topmanager $\mathbf{2 0 0 5}-$ in \% - } \\
\hline & F & GB & ESP & D & IT & SWE \\
\hline Großbürgertum & 57,0 & 53,2 & 55,0 & 51,7 & 51,6 & 28,6 \\
Bürgertum & 30,3 & 31,2 & 30,0 & 33,3 & 16,1 & 21,4 \\
Mittelschichten/Arbeiterklasse & 12,7 & 15,6 & 15,0 & 15,0 & 32,3 & 50,0 \\
\hline & & & & & WS I MITTEILUNGEN \\
Quelle: Recherchen des Autors. & & & &
\end{tabular}

lich offener dagegen ist die Wirtschaftselite in den meisten anderen Staaten, vor allem in den skandinavischen. So stammt zum Beispiel in Schweden jeder zweite Spitzenmanager aus der breiten Bevölkerung und nur jeder vierte aus dem Großbürgertum (Tabelle 3).

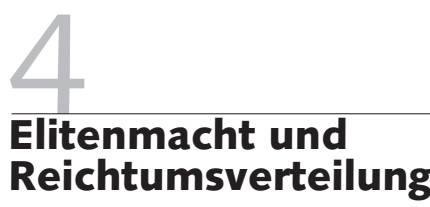

Da die Verteilung des gesellschaftlichen Reichtums in der Regel der beste Indikator für die Macht der Eliten ist, müsste die Hierarchie der Elitetypen weitgehend parallel zu einer Hierarchie der nationalen Einkommensunterschiede verlaufen. Das bestätigt sich im Großen und Ganzen auch. Es gibt in puncto Einkommensungleichheit ebenfalls vier klar voneinander getrennte Gruppen. Die geringsten Einkommens- wie Lohndifferenzen zwischen oben und unten und die niedrigsten Armutsquoten weisen die skandinavischen Länder auf. Mit Tschechien und Ungarn folgen zwei ehemalige Ostblockländer relativ dicht auf. Dann kommt ein breites Mittelfeld, zu dem mit Ausnahme der britischen Inseln alle traditionellen EU-Staaten zählen. Großbritannien und Irland bilden zusammen mit den südeuropäischen Ländern die Schlussgruppe (Tabelle 1).

Vergleicht man diese vier Ländergruppen mit den vier Elitetypen, so fallen die Parallelen sofort ins Auge. Auf der einen Seite des Spektrums stehen die skandinavischen Staaten. Sie haben die sozial durchlässigsten und inhomogensten Eliten und weisen gleichzeitig das geringste $\mathrm{Ma}$ an sozialer Ungleichheit auf. Auf der anderen Seite stehen Großbritannien, Spanien und Portugal. Ihre Eliten zeichnen sich durch eine hohe soziale Herkunft und große Homogenität aus. Die Spaltung zwischen Reich und Arm fällt dann auch erwartungsgemäß sehr scharf aus. Dazwischen liegt ein breites Mittelfeld. Es wird sowohl in puncto Elitenherkunft und -homoge- nität als auch in Bezug auf Einkommensdifferenzen und Armutsquoten von Deutschland angeführt.

Erklärungsbedürftig sind allerdings die Ergebnisse für Italien und vor allem Frankreich. Italien fällt aus dem Rahmen, weil die Kluft zwischen Reich und Arm deutlich tiefer ist als es angesichts der Elitenstruktur zu erwarten gewesen wäre. Bei Frankreich ist es genau umgekehrt. Beide Fälle zeigen, dass sich im Großen und Ganzen zwar eindeutige Parallelen zwischen Elitentypus und dem Umfang der gesellschaftlichen Ungleichheit ziehen lassen, man dabei aber nie die jeweiligen Traditionen der einzelnen Länder und die dort herrschenden Kräfteverhältnisse ignorieren darf.

In Italien sind es in erster Linie zwei Faktoren, welche die doch überraschend große Kluft zwischen Reich und Arm erklären. Das ist zum einen der für ein entwickeltes Land mit $30 \%$ der Erwerbstätigen sehr hohe Anteil an kleinen, schlecht verdienenden Selbstständigen; zum anderen ist es die schlechte finanzielle Absicherung vieler Arbeitsloser. Die durchschnittliche Lohnersatzquote, d.h. Höhe und Dauer von Arbeitslosengeld und Sozialhilfe verglichen mit den Nettoeinkommen, liegt in Italien mit $6 \%$ weit unterhalb des europäischen Durchschnitts von gut $70 \%$ (Jörgensen/ Schulz zur Wiesch 2006, S.14).

Dass Frankreich eine weit ausgeglichenere Einkommensverteilung aufweist als die beiden Länder Europas, die ihm in puncto Elitenherkunft und -homogenität am ähnlichsten sind - Großbritannien und wöhnlich starken Staatsorientierung der Bevölkerung, von der auch die Eliten profitieren. Die Staatsorientierung bildet nicht nur die Grundlage des extrem zentralisierten Elitebildungssystems, d.h. letztlich die Basis der hohen Homogenität wie Mobilität der französischen Eliten, sie verleiht ihnen auch öffentliche Legitimität, wie Bourdieu in seinen Schriften immer wieder betont (Bourdieu 2004).

Die für die Eliten ideologisch wie materiell sehr vorteilhafte Staatsorientierung hat aber auch eine Kehrseite. Die BevölkeSpanien -, liegt vor allem an der außerge- rung fordert die damit einhergehenden Ansprüche an den Staat in bestimmten Abständen auch immer wieder handfest ein, wie z. B. beim großen Streik 1995 gegen die Rentenpläne der damaligen Regierung Juppé. Der Staat ist zwar der entscheidende Adressat der Forderungen, seine Spitzenvertreter wie auch die Eliten ganz allgemein werden aber sehr misstrauisch beäugt. Daher kommt es regelmäßig zu massiven Konflikten, die sich in Generalstreiks, groBen Demonstrationen und teilweise auch gewalttätigen Unruhen ausdrücken. Durch sie werden den Plänen der Eliten immer wieder Grenzen gezogen, wie z. B. im letzten Jahr beim Versuch der Regierung de Villepin, den Kündigungsschutz für Jugendliche weitgehend aufzuweichen. Die latente und auch immer wieder manifest werdende Protestbereitschaft erheblicher Teile der Bevölkerung engt den Spielraum für extreme Einkommensdifferenzen nach angelsächsischem Muster spürbar ein.

Wie stark dieser Faktor die Einkommensverteilung beeinflusst, zeigt ein Vergleich zwischen Deutschland und Frankreich. In beiden Ländern gelangten in der zweiten Hälfte der 1990er Jahre „linke“ Koalitionen an die Regierung, 1997 unter Jospin und 1998 unter Schröder. Die Folgen für die Einkommensverteilung waren aber höchst unterschiedlich. Die Steuer- und Sozialpolitik der rot-grünen Berliner Regierung verschärfte die Einkommensungleichheit in Deutschland ganz massiv. Die Differenz zwischen den Einkommen des obersten und des untersten Fünftels der Bevölkerung stieg zwischen 1998 und 2004 mit über $20 \%$ mehr als doppelt so stark an wie in jedem anderen Land Westeuropas, und das schon vor dem Inkrafttreten von Hartz IV. Sie erreichte damit einen Wert, wie er in Frankreich vor dem Regierungsantritt Jospins 1997 bestand. In Frankreich ging die Einkommensdifferenz bis 2002 dagegen sogar um ca. $10 \%$ zurück, sodass sie - auch wenn unter Raffarin, dem konservativen Nachfolger Jospins, eine leichte Gegenbewegung einsetzte-2004 immer noch geringer war als 1997. Und was noch bemerkenswerter ist: Die Einkommensdifferenz in Frankreich war damit niedriger als in Deutschland. Der große Streik von 1995 wirkte in dieser Hinsicht spürbar nach. Zwar sorgt der schwache gewerkschaftliche Organisierungsgrad in Frankreich für große Gehalts- und Lohnunterschiede ( $\mathrm{Ta}$ belle 1); in Bezug auf die Gesamteinkommen wird das aber durch die relativ umfas- 
senden staatlichen Sozialleistungen mehr als wettgemacht. Das französische Beispiel demonstriert unübersehbar, wie wichtig nationale Traditionen sind. Die Macht, die die nationalen Eliten auszuüben vermögen, hängt stark von den Traditionen ab; denn sie prägen nicht nur die spezifisch nationalen Typen von Elitebildung und damit die Rekrutierung und Homogenität der jeweiligen Eliten; sie prägen vielmehr genauso auch die Einstellung und das Verhalten der Bevölkerung diesen Eliten gegenüber, d.h. die Stärke potenzieller Gegenkräfte.

Die relativ geringen sozialen Differenzen in den skandinavischen Ländern lassen sich nicht ohne die lange egalitäre Tradition der skandinavischen Gesellschaften, die Tradition des Wohlfahrtsstaates, die Dominanz der sozialdemokratischen Parteien und die Stärke der Gewerkschaften erklären. Die egalitäre Grundeinstellung der überwiegenden Bevölkerungsmehrheit, eine unter besonderen historischen Bedingungen entstandene Mischung aus bäuerlichen und proletarischen Elementen, zeigt sich in vielen Aspekten des täglichen Lebens. Zwar verliert diese Tradition auch in Skandinavien an Bedeutung, sie prägt die Gesellschaften aber immer noch stark. Das hat die neue konservative Regierung Schwedens leidvoll erfahren müssen. Wegen nicht gezahlter Rundfunk- und Fernsehgebühren und der Schwarzbeschäftigung von Haushaltshilfen mussten bereits kurz nach Regierungsantritt zwei Ministerinnen zurücktreten.

Auch der Wohlfahrtsstaat genießt in Skandinavien nach wie vor eine große Wertschätzung. Die für seine Finanzierung notwendigen, im internationalen Vergleich außergewöhnlich hohen Einkommenssteuern werden von der überwiegenden Mehrheit der Bevölkerung bis heute akzeptiert. Die großen Erfolge der skandinavischen Bildungssysteme, die es im Unterschied gerade zum deutschen Bildungssystem schaffen, die Bedeutung der sozialen Herkunft für die Bildungskarrieren deutlich zu reduzieren und einen großen Prozentsatz der Bevölkerung zu hohen Bildungsabschlüssen zu führen, passen ebenfalls ins Bild. Zu guter Letzt sind auch die skandinavischen Gewerkschaften ausgesprochen erfolgreich. Sie sind nicht nur diejenigen in Europa mit dem weitaus höchsten Organisationsgrad (zwischen 60 und $80 \%$ ); sie gehören auch zu den ganz wenigen, die in den letzten zwei Jahrzehnten keinen spürbaren Rückgang zu ver- zeichnen hatten (Carley 2004; Ebbinghaus/Visser 2000; Schnabel/Wagner 2005). Die im europäischen Vergleich hohe soziale Durchlässigkeit der Eliten in diesen Ländern ist das Produkt dieses spezifisch skandinavischen Modells, trägt aber auch zu seiner Stabilität und Kontinuität bei.

Man darf dabei allerdings zwei Punkte nicht übersehen. Zum einen ist die politische Aktivität der breiten Bevölkerung auch in den skandinavischen Ländern in den letzten zwei Jahrzehnten deutlich zurückgegangen. Die traditionell außerordentlich mitgliederstarken Parteien haben seit 1980 zwischen gut einem Viertel und ungefähr der Hälfte ihrer Mitglieder verloren, obwohl sie immer noch einen mehr als doppelt so hohen Anteil der Bevölkerung organisieren wie etwa in den Niederlanden oder Deutschland (Lucardie 2006, S. 347; Steffen 2006, S. 97). Zum anderen sind die Veränderung der Kräfteverhältnisse zulasten der breiten Bevölkerung und der Siegeszug neoliberalen Gedankenguts auch an Skandinavien nicht spurlos vorübergegangen. Die letzte sozialdemokratische Regierung in Schweden hat die Erbschaftssteuer weitgehend abgeschafft, die neue konservative Regierung will auch die Vermögenssteuer abschaffen. Die Unternehmenssteuern sind ebenfalls schon unter den Sozialdemokraten spürbar gesenkt worden, liegen real allerdings mit gut $33 \%$ immer noch deutlich über dem EU-Durchschnitt von ca. $25 \%$ (European Commission 2007, S. 92, 219). Auch angesichts der sehr hohen Einkommenssteuersätze gerade für Spitzenverdiener, die in Dänemark in den letzten Jahren sogar noch einmal auf jetzt $59 \%$ angehoben worden sind, ist all das zwar nicht so problematisch wie die Steuersenkungen in Deutschland oder gar den angelsächsischen Ländern; es zeigt jedoch, dass kein Modell auf ewig existieren muss. Das gilt für den Wohlfahrtsstaat genauso wie für die Rekrutierung und Homogenität der Eliten. Wie eine Veränderung der gesellschaftlichen Kräfteverhältnisse den Prozess der Elitenbildung beeinflusst, kann man in den letzten Jahren in Deutschland und Italien sehen.

\section{Ausblick}

Die augenblickliche Entwicklung muss eher pessimistisch stimmen. Der fast über- all zu beobachtende, vielfach dramatische Rückgang der Gewerkschaftsmitglieder, die massiv gesunkene Bereitschaft der breiten Bevölkerung zum politischen Engagement, die auf fast allen Ebenen wachsende Macht der großen Unternehmen, die zunehmenden Lobbyaktivitäten bei den nationalen Regierungen wie bei der EU in Brüssel sprechen nicht gerade für eine Verbesserung der Situation, soweit es die Lebenslage der breiten Bevölkerung oder ihre Einflussmöglichkeiten angeht.

Die Lage wird noch dadurch verschärft, dass die zentralen Instanzen der EU-Bürokratie nur in vergleichsweise geringem Maße dem demokratischen Einfluss seitens der Bevölkerungen der EU-Mitgliedsstaaten unterliegen. Die Europäische Kommission wie auch die hohen Beamten sind für die breite Bevölkerung noch weniger greifbar als die nationalen Regierungs- und Verwaltungsspitzen, beeinflussen aber mit ihren Entscheidungen das normale Leben in Europa immer stärker. Nur mit länderübergreifenden massiven Protesten konnten in den letzten Jahren Pläne der EUKommission zum weiteren Abbau sozialer Leistungen und Rechte verhindert werden. Solche Erfolge oder zumindest Teilerfolgewie bei dem erfolgreichen Streik der Hafenarbeiter gegen den unter dem Namen „Port Package II“ bekannt gewordenen Plan, die Lohn- und Sozialstandards in den Häfen zu senken, oder bei den großen Demonstrationen gegen die Bolkestein-Richtlinie - waren jedoch die Ausnahme. In der Regel wurden die (vielfach unter erheblichem Einfluss der Unternehmenslobby verfassten) neuen Richtlinien verabschiedet und dann in nationales Recht umgesetzt. Die überwiegend bürgerlich-großbürgerliche Herkunft der meisten EUKommissare und der nationalen Eliten der wichtigen EU-Staaten erleichtert dabei die Verständigung über die zentralen Ziele deutlich.

Wer glaubt, auf die Einsicht dieser Eliten setzen zu können, dürfte in den allermeisten Fällen enttäuscht werden. Ohne massiven Druck seitens der Bevölkerung wird sich an dem augenblicklichen Kurs, der die sozialen Unterschiede in Europa weiter verschärft, nicht viel ändern. Die von der Bundesregierung jüngst beschlossenen weiteren Steuerentlastungen für die Wirtschaft belegen das unmissverständlich. Warum sollten die Eliten auch etwas ändern? Sie gehören ja zu den Gewinnern der Entwicklung. 
Bourdieu, P. (2004): Der Staatsadel, Konstanz

Bundesministerium der Finanzen (BMF) (2005): Die wichtigsten Steuern im internationalen Vergleich 2005, Berlin

Bundesministerium der Finanzen (BMF) (2007): Die wichtigsten Steuern im internationalen Vergleich 2006, in: Monatsbericht des BMF - Januar 2007, Berlin, S. 53-68

Bundesministerium für Gesundheit und Soziale Sicherung (BMG) (2005): Lebenslagen in Deutschland. Der 2. Armuts- und Reichtumsbericht der Bundesregierung, Kurzfassung, Berlin

Carley, M. (2004): Trade Union Membership 1993-2003, eurofound. europa.eu/eiro/2004/03

Dunkel, M. (2007): Deutschlands ungerechte Steuerreformen, in: Financial Times Deutschland vom 1.3.

Ebbinghaus, B. /Visser, J. (2000): Trade Unions in Western Europe since 1945, London

European Commission (2005): Employment in Europe 2005, Brüssel European Commission (2007): Taxation trends in the European Union. Data for the EU Member States and Norway. Eurostat Statistical Books, Luxemburg

Grabka, M. M./Frick, J. R. (2007): Vermögen in Deutschland wesentlich ungleicher verteilt als Einkommen, in: DIW-Wochenbericht 45, S. 665672

Groh-Samberg, O. (2007): Armut in Deutschland verfestigt sich, in: Wochenbericht des DIW Berlin 12, S. 177-182
Guio, A.-C. (2005): Einkommensarmut und soziale Ausgrenzung, in: EU25. Statistik kurz gefasst, Bevölkerung und soziale Bedingungen 13, Brüssel

Hartmann, M. (2007): Eliten und Macht in Europa, Frankfurt/Main Jörgensen, J. F./Schulz zur Wiersch, J. (2006): Wie sozial ist Europa? Eine Kurzstudie zur Sozialen Lage in der EU, Berlin Liddle, R./Lerais, F. (2006): Europe's Social Reality. A Consultation Paper from the Bureau of European Policy Advisers, Brüssel

Lucardie, P. (2006): Das Parteiensystem der Niederlande, in: Niedermayer, O. et al. (Hrsg.): Die Parteiensysteme Westeuropas, Wiesbaden, S. 67-107

Mattheis, H. (2007): Nach riesigem Vermögenszugewinn: für mehr Verteilungsgerechtigkeit - Kein weiterer Bonus für Superreiche!, Pressemitteilung der SPD vom 19.10.

Schnabel, C./Wagner, J. (2005): Determinants of Union Membership in 18 EU Countries: Evidence from Micro Data, 2002/03. Discussion Paper 31, Lehrstuhl für VWL, Friedrich-Alexander-Universität Erlangen-Nürnberg

SPD-Bundestagsfraktion (2007): Reichtum in Deutschland. Konferenz der Arbeitsgruppe Verteilungsgerechtigkeit und soziale Integration der SPD-Bundestagsfraktion am 23.10.2006 in Berlin

Steffen, C. (2006): Die Parteiensystems Dänemarks, Norwegens und Schwedens, in: Niedermayer, O. et al. (Hrsg.): Die Parteiensysteme Westeuropas, Wiesbaden, S. 67-107 